\title{
Why Should We Have a Periodic Safety and Performance Program for Medical Devices
}

\author{
Tavakoli Golpaygani A. ${ }^{*}$
}

\begin{abstract}
Nowadays, more than 10,000 different types of medical devices can be found in hospitals. These devices used in medical centers and hospitals for monitoring and treatment of patients require periodic safety and performance checking in order to have confidence in their functioning and operation. Physicians need better accurate medical measurements in order to better diagnose diseases, monitor patients and deliver treatments, in this way failure to ensure appropriate measurements will certainly have diverse effects. Safety and performance testing of medical devices in the medical sector is a one of the key factor in improving public health. Acquiring results of some investigations indicate a need for new and severe regulations on periodic performance verifications and medical equipment quality control program especially in highrisks instruments.

The metrological reliability of four high risk medical devices, Electrosurgical unit, Defibrillator, Syringe pump and Infant incubator in use some hospitals (privates and publics) in one of the province of Iran according to international and national standards was evaluated. Quantitative analysis of Some parameters that impact the safety and performance showed the amount of the obtained results in some equipment are in critical range and have higher values than standard limitations. General electrical safety evaluations for measuring the patient leakage currents and patient auxiliary currents carried out for all of groups, in some cases the amount of leakage currents were over the standard limitations.

Acquiring results indicate a need for new and severe regulations on periodic performance verifications and medical equipment quality control program especially in high risk instruments. It is also necessary provide training courses for operating staff in the field of meterology in medicine and what's the critical parameters and how they can get good accuracy results equipment.
\end{abstract}

\section{Keywords}

Metrology, Medical devices, Safety, Performance, Calibration

\section{Introduction}

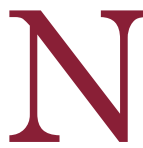

owadays, electrical devices are being employed in a wide variety of fields in medical sciences with different effects. According to the importance of this matter in relation to human health, periodic verifications, safety and performance testing of medical devices in the form of quality control tests and calibration are necessary. This issue has started and noticed seriously since the past decade for medical devices.

Even the best medical measuring instruments and equipment are affected by wearing and tearing, environmental changes and other fac-
${ }^{1}$ Department of Biomedical Engineering, Standard Research Institiute, Karaj, Iran

Corresponding author:

A. Tavakoli Golpaygani

Faculty of Electrical, Mechanical and Construc-

tion Engineering,

Standard Research Insti-

tute, Karaj, Iran

E-mail: tavakoli.golpa@ gmail.com

Received: 14 April 2017 Accepted: 20 July 2017 
tors, losing accuracy over time. According to the importance to human health, systematic and periodic checking of test and measuring instruments, national and international standards on a regular schedule are very essential for reliable measurements. This process has many advantages such as maximizing effectiveness and minimizing risk for patients and operators.

Safety and performance testing are required to ensure the results and reports which are accurate, reliable and safe to deliver treatment and diagnose process. The accuracy of the instrument readings provides how the medical devices can generate test results which are very close to the true value. For example, in many illnesses such as hypertension symptoms; a diagnostic process starts with only one physiological parameter (blood pressure) measurement. In the other side in many situations, the initial stage of disease diagnosis starts with laboratory testing of different samples from patients (such as blood, urine, ...) ,then, the laboratory output is only some analytical data. These data are used in determining whether a patient has a disease or not. We expect a high quality service from every clinical laboratory, which all test results produced by all laboratories at all times should be accurate and clinically meaningful. Medical error measurements made by the medical devices tend to shift the test results from the true value. There forean error can cause under-detection or over-detection of diseases. Physicians decided based on the clinical results obtained from the different measurements, if the results are not correct and accurate, it might cause the physician to under-dose or over-dose the patient with incorrect prescriptions. [1,2].

\section{Material and Methods}

Recently, it has been seen from 5,000 to more than 10,000 different types of medical devices in an average to large sized hospitals. Hospitals and medical centers must ensure that their critical medical devices are safe, accurate, reliable and operating at the required level of performance. For instance the results of four investigations of electrical safety and metrological reliability for four important medical devices with a high applicability in treatment process are presented. In each of these studies at least twenty equipment with different models were used at some publics and private hospitals were tested. A schematic of each device is presented in Figure 1.

\section{A Case study on Electrosurgical Equipment}

Electrosurgical units (ESU) are the most common type of electrical equipment in the operating room. Electrosurgical unit is one of the most effective surgical instruments, through the better control and the management of complications during surgery, it improved patient safety. The principle of electrosurgery is inducing heat by high frequency electrical currents. Coagulation, cutting, desiccation and fulguration of biological tissue are different effects of electrosurgery function. The correct operation of electrosurgical unit is essential to ensure patient safety. In this case study, quantitative analysis of HF leakage current measurements, showed the amount of the obtained results in many units is in critical ranges and has higher values than standard limitations especially in ground referenced generators.

The amount of earth resistance of hospitals or medical centers has important effects on HF leakage currents of electrosurgical equipment. High earth resistance causes high HF leakage current, as a result, the quality of earth system of medical centers has a key role in safety performance of an electrosurgical unit. The achieved results in this case study represent that HF leakage current of ground referenced generators are more than isolated generators. Therefore, the use of isolated generators in order to reach to high level of patients' safety is preferred in medical centers [3]. 


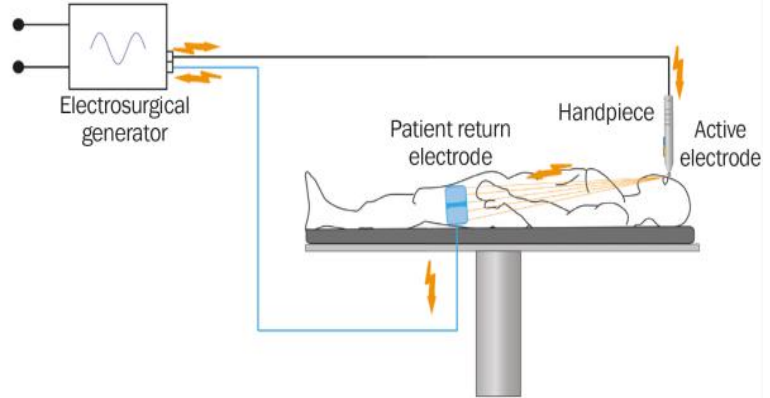

(a) Electrosurgical Unite

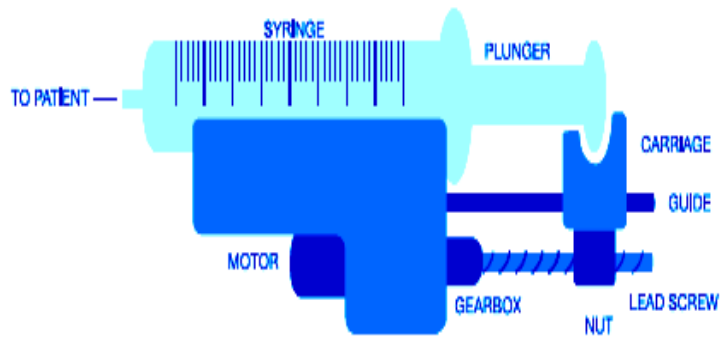

(c) Syringe pump

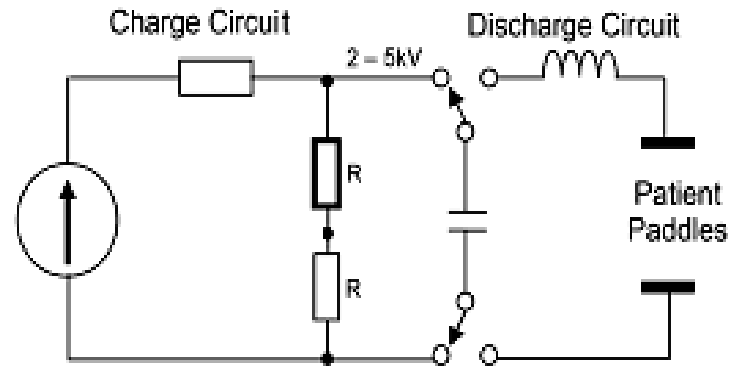

(b) Defibrillator Circuit

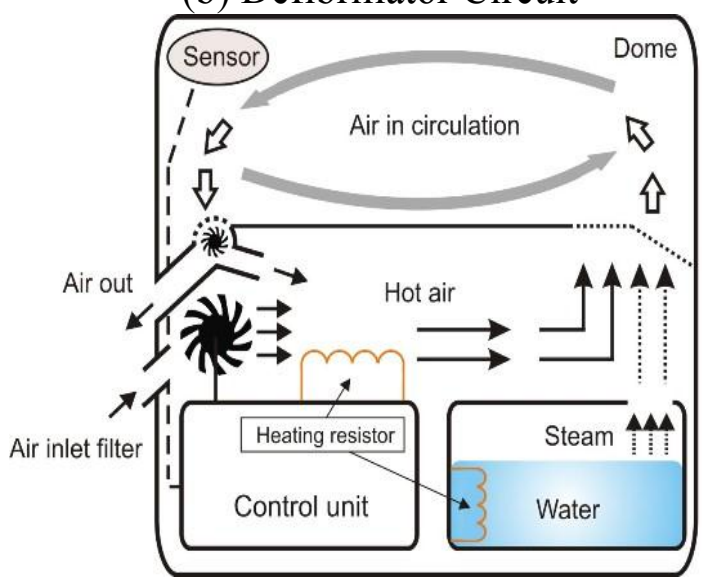

(d) Infant Incubator

Figure 1: A schematic of medical devices in case studies

\section{A Case Study on Defibrillators Equipment}

Medical research and statistical analysis all over the world show generally over 135,000 people die annually following acute myocardial infarctions as ventricular fibrillation (VF) or ventricular tachycardia (VT), and the only effective treatment for which is early defibrillations. Defibrillation is the application of a predefine electrical current through the myocardium, this phenomenon causes synchronous depolarization of the cardiac muscle. This action terminates the arrhythmia, and allows normal sinus rhythm to be reestablished.

In this case, study on the performance and safety testing of frequent use, manual defibril- lators equipment in the use at some hospitals were evaluated. Quantitative analysis of accuracy of delivered energy measurements, represented the amount of the obtained results in many units are critical and have less value over the standard limitations, especially in devices with poor quality battery. The delivered energy of defibrillators especially in frequent discharge is dependent seriously on the power battery condition. Only some devices delivered acceptable output accuracy of delivered energy and the accuracy in poor quality battery conditions, after activation of discharge alarm were low.

General electrical safety evaluations for measuring the patient leakage current and pa- 
tient auxiliary leakage current carried out for all of the under test devices. In some cases the amount of leakage currents were over the standard limitations. As a technical investigation, it is shown that the earth system quality of hospital has a role key in electrical safety test and it is necessary to evaluate the quality of hospital earth system [4].

\section{A Case Study on Infusion Pumps Equipment}

Infusion devices are used extensively in clinical settings and patients' homes as essential tools to provid critical care, perioperative care and management of pain. It is estimated that $80 \%$ of hospitalized patients receive intravenous (IV) therapy. Because infusion pumps are frequently used to administer critical fluids, including high-risk medications, pump failures can have significant implications for patient safety. Hence, the reliability of infusion pumps is extremely important. In the third study, quantitative analysis of flow rate accuracy measurements and represented the amount of the obtained results in some units are critical and have inaccurate values, especially in equipment with inappropriate IV set. For example, it has been seen, the usage of a wrong or nonstandard syringe or tubing set, which is not made based on the approved technical specifications, increases the occurred error percent and the inaccuracy 10 to 20 percent. The position of drip detector is an important factor in infusion pumps. If the detector is installed near the injection location, then the error percent can increase up to $25 \%$. The reduction of battery power is one of the effective factors on the performance of the infusion pumps. It has been seen that the low quality batteries and the batteries which are sensitive to the improper voltage could reduce the outflow rate between 10 to 30 percent.

General electrical safety evaluations for measuring the patient leakage current and pa- tient auxiliary leakage current carried out for all of the under test devices. In some cases, the amount of leakage currents was over the standard limitations.

Due to design and manufacturing technology development of the pumps, it is required to consider supplementary professional training courses for the users to enable them to use all available pump options and reduce the human errors [5].

\section{A Case Study on Infant Incubators Equipment}

Today's, the most of the neonatal or newborn babies were put in infant incubators for overcoming the distress during the post-natal care. These incubators can be defined as an optimal cabin environment with electronic equipment supplying the premature or babies with illness problems to be able to continue their vital functions. It shows more than $80 \%$ of neonatal babies are hospitalized in infant incubators and are treated there over a period of 3 days to 7 months. In the fourth study, a quantitative analysis of temperature, humidity accuracy measurements, the stability of temperature, represented the amount of the obtained results in some units are critical and are not in standard limitations. Stability of temperature is an important factor, some incidents such as apnea can result from variations in the incubator temperature. In some devices with repair history have been seen that selected and replaced temperature sensors have not had required accuracy as defined specifications in manufacture's documents or manual.

General electrical safety evaluations for measuring the patient leakage currents and patient auxiliary currents were carried out for all of the under test devices. In some cases the amount of patient leakage currents was over the standard limitations, especially in two under test devices with recoiling of motor's fan, the patient leakage current and the noise level 
of fan were more than limited amounts [6].

\section{Discussion}

Safety and performance testing of medical devices in the medical sector is a one of the key factor in improving public health. For the best confidence in their functioning and operation, the medical devices used in medical centers and hospitals for monitoring and treatment of patients require periodic safety and performance checking. Physicians need better accurate medical measurements in order to diagnose diseases better, monitor patients and deliver treatments.

Even an error in a simple medical device such as clinical thermometers to measure body temperature, sphygmomanometers to measure blood pressure, weighing scales to measure body weight etc. can cause medical decision making to shift from the normal decision. In some condition, medical errors might cause patients pain bearing permanent or temporary disability and in some state even cause death. In another view, this matter can cause increasing health costs. Increasing the number of follow-up tests, medication and treatment for a patient who has been incorrectly diagnosed to have a certain disease will be incurred health costs.

\section{Conclusion}

Acquiring results in four discussed case studies indicate a need for new and severe regulations on periodic performance verifications and medical equipment quality control and calibration. This matter is more significant in high-risks medical devices. In many hospitals, there are some deficiencies related to the operator and users of medical devices, some of them are noticed below.

1. They thought that a calibrated device works without any errors for a long time and no need recalibration in defined intervals.

2. The calibration certificates are kept in the outside of the operating area and difficult to access for operators and users.

3 . The benefits of a calibration service is unknown and in many situation is regarded as a purchased document.

So, it is also necessary to provide training courses on the fundamental of operation and performance parameters for medical staff in the field of metrology in medicine and how they can get good accuracy results especially in high risk medical devices.

Finally, it is obvious, responsibility, education, commitment, management support and sufficient resources are the strongest drivers for developing the successful culture of maintenance and calibration in technical medical management.

\section{Acknowledgment}

The author would like to express his thanks to all experts participated in the study from biomedical engineering department of hospitals and medical centers.

\section{Conflict of Interest}

None

\section{References}

1. Khandpour RS. Handbook of Biomedical Instrumentation. New York: Mc Graw Hill; 2014.

2. Noblitt J. Introduction to Biomedical Engineering Technology. Biomed Instrum Technol. 2008;42:446.

3. Tavakoli Golpaygani A, Movahedi MM, Reza M. A Study on Performance and Safety Tests of Electrosurgical Equipment. $J$ Biomed Phys Eng. 2016;6:175-82. PubMed PMID: 27853725; PubMed Central PMCID: PMC5106550.

4. Tavakoli Golpaygani A, Movahedi M, Reza $M$, Hassani K. A study on performance and safety test of infusion pump devices. 2017. 
5. Tavakoli Golpaygani A, Movahedi M, Reza M, Hassani K. A study on performance and safety test of infusion pump devices. Biomedical Research. 2017;28.
6. Tavakoli Golpaygani A., Movahedi M.M. and Reza M."A study on performance and safety test of infant incubators devices" $J$. Of Clinical Engineering. 2018, In Press. 